\title{
Comunicación
}

\section{Temperatura ambiental y su efecto sobre parámetros fisiológicos en vacas Nellore y cruces bajo condiciones del trópico peruano}

\section{Environmental temperature and its effect on physiological parameters in Nellore cows and crosses under conditions of the Peruvian tropics}

\author{
Ide Unchupaico P. ${ }^{1,4}$, Luis Bazán A. ${ }^{2}$, Carlos Quispe E. ${ }^{3}$, Edith Ancco G. ${ }^{3}$
}

\section{Resumen}

El objetivo del estudio fue determinar las constantes fisiológicas relacionadas con la termorregulación corporal, así como parámetros hematológicos básicos entre vacas Nellore y sus cruces bajo estrés calórico. El estudio se desarrolló en la provincia de Satipo, región Junín, Perú, a 628 msnm. Se trabajó con 16 vacas cruzadas (Brown Swiss x Nellore) y 16 vacas Nellore. Las constantes fisiológicas (temperatura corporal, frecuencia respiratoria y frecuencia cardiaca) se evaluaron en horas de la mañana (06:00-07:00) y en la tarde (14:00-17:00). Se tomaron muestras de sangre para determinar hemoglobina (g/dl), hematocrito (\%), volumen corpuscular medio (VCM, fl), hemoglobina corpuscular media (HCM, pg), concentración de hemoglobina corpuscular media (CHCM, g/dl). Las funciones termorreguladoras (temperatura rectal, frecuencia cardiaca y frecuencia respiratoria) se vieron afectadas en horas de estrés calórico en los dos grupos raciales $(p<0.05)$. Los valores hematológicos se encontraron dentro de los rangos referenciales, excepto el VCM y la HCM.

Palabras clave: estrés calórico, vacas, hematología, termorregulación, parámetros fisiológicos

${ }^{1}$ Facultad de Zootecnia, Universidad Nacional del Centro del Perú, Junín, Perú

${ }^{2}$ Escuela Profesional Zootecnia Tropical, Facultad de Ciencias Agrarias, Universidad Nacional del Centro del Perú, Junín, Perú

${ }^{3}$ Escuela Profesional de Medicina Veterinaria y Zootecnia, Facultad de Ciencias de la Salud, Universidad Peruana los Andes, Junín, Perú

${ }^{4}$ E-mail: iunchupaico@uncp.edu.pe

Recibido: 1 de abril de 2019

Aceptado para publicación: 23 de enero de 2020

Publicado: 31 de marzo de 2020 
The aim of this study was to determine the physiological parameters related to body thermoregulation, as well as basic haematological parameters between Nellore cows and crossbreds under heat stress. The study was carried out in the province of Satipo, Junín region, Peru, at 628 meters above sea level. Sixteen crossbred cows (Brown Swiss x Nellore) and 16 Nellore cows were used. The physiological parameters (body temperature, respiratory rate and heart rate) were evaluated in the morning (06:00-07:00) and in the afternoon (14:00-17:00). Blood samples were taken to determine haemoglobin $(\mathrm{g} / \mathrm{dl})$, haematocrit $(\%)$, mean corpuscular volume $(\mathrm{MCV}, \mathrm{fl})$, mean corpuscular haemoglobin ( $\mathrm{MCH}, \mathrm{pg}$ ), mean corpuscular haemoglobin concentration ( $\mathrm{MCHC}, \mathrm{g} / \mathrm{dl}$ ). Thermoregulatory functions (rectal temperature, heart rate and respiratory rate) were affected during hours of heat stress in both racial groups $(p<0.05)$. Haematological values were found within the referential ranges, except the $\mathrm{MCV}$ and $\mathrm{MCH}$.

Key words: heat stress, cows, haematology, thermoregulation, physiological parameters

\section{INTRODUCCIÓN}

El manejo ganadero en climas tropicales debe trabajarse en interacción con el medio ambiente, especialmente en lo relacionado con las consecuencias del estrés calórico (A raúz et al., 2010), siendo importante planificar estrategias que permitan maximizar la productividad de los animales, especialmente en sistemas semi extensivos. Roca (2011) menciona que el estrés en bovinos de leche es provocado por una alta tasa de calor endógeno causado por las altas temperaturas y humedad relativa del ambiente externo que el organismo del animal no es capaz de disipar por medio del sistema de regulación térmica.

Los primeros efectos ante un estrés térmico en los animales es una disminución en el consumo de materia seca y un aumento del consumo diario de agua, los cuales tienen un impacto directo sobre la regulación de la temperatura corporal (Arias y Mader, 2011). Además, el aumento en el consumo de agua incide sobre el volumen sanguíneo. En las primeras fases de los procesos de termorregulación se produce taquicardia y vasodilatación cutánea con el fin de reducir el calor corporal a través de la piel (Klein, 2014). Las razas más susceptibles al estrés térmico son las del género Bos taurus (Espinoza et al., 2009), mientras que el ganado Bos indicus presenta una composición genética que le confiere una mayor termotolerancia, lo cual les hace más adaptables a climas de calor (Hansen, 2004). Fisiológicamente, estas razas tienen una mayor capacidad para transferir el calor interno al exterior y regular con mayor eficiencia la temperatura corporal, mientras que los cruces Bos taurus x Bos indicus pueden adquirir capacidades intermedias de adaptación; sin embargo, hay pocos estudios que reporten esas habilidades ante situaciones de estrés térmico.

Se dispone de diversas variables hematológicas que pueden ser usadas como indicadores adicionales de estrés térmico. Estas variables han sido medidas en aves (Díaz et al., 2016) y en bovinos de carne y leche y en diversas condiciones. Se explica que las variaciones hematológicas tienen su origen en mecanismos de adaptación fisiológica relacionados con un incremento en las necesidades de oxigenación para activar las vías de termorregulación (Ribeiro et al., 2018). Así mismo, estarían asociadas a un incremento hormonal, entre las que se en- 
cuentra el cortisol, las catecolaminas y la tiroxina (Klein, 2014). Esta circunstancia fisiológica hace que la tasa de oxigenación a nivel celular se vea elevada, de manera que se incrementan diversos parámetros hematológicos para garantizar la eficiencia en el transporte del oxígeno (Olkowski et al., 2005).

Se requieren estudios adicionales de otros parámetros hematológicos como la hemoglobina corpuscular media (HCM), el volumen corpuscular medio (VCM) y la concentración de hemoglobina corpuscular media $(\mathrm{CHCM})$ que estén relacionados al estrés térmico.

Bajo ese contexto es importante caracterizar la producción pecuaria bajo el efecto de las altas temperaturas a fin de plantear estrategias acordes con el medio a fin de mitigar posibles efectos nocivos. Un indicador del bienestar animal son las características termorreguladoras, los parámetros hematológicos y el consumo voluntario, las cuales pueden relacionarse con los efectos climáticos, de allí su importancia de conocer como estas variables se ven afectadas por el estrés calórico. El objetivo general del estudio fue determinar las funciones termorreguladoras, hematología básica y la comparación entre vacas de la raza Nellore y sus cruces con Brown Swiss sometidas a pastoreo extensivo con marcados episodios de estrés calórico.

\section{Materiales y Métodos}

El estudio se desarrolló en la Estación Experimental Agropecuaria de la Universidad Nacional del Centro del Perú, en la provincia de Satipo, región Junín, Perú, a 628 msnm. La población fue de 92 vacas y se utilizaron 16 vacas cruzadas (Brown Swiss $\mathrm{x}$ Nellore) y 16 vacas Nellore. Se escogieron únicamente animales con 2 a 4 partos, en segundo tercio de lactación, preñadas y con cría al pie. Las 32 vacas del estudio estuvieron sometidas a un manejo constante; sin embargo, las vacas Nellore eran menos dóciles que las cruzadas. El pastoreo se estableció entre las 07:00 hasta las 16:00 horas, permaneciendo durante la noche en un corral con sombra. Todas las variables fueron registradas en un brete cercano al potrero de pastoreo.

Se registró la temperatura corporal $\left({ }^{\circ} \mathrm{C}\right)$, frecuencia respiratoria (ciclos respiratorios / minuto), frecuencia cardiaca. (latidos/minuto) en dos horarios entre las 06:00 y 07:00 $\left(18{ }^{\circ} \mathrm{C}\right.$ de temperatura ambiental y $80 \%$ de humedad relativa) y entre las 14:00 y 15:00 horas $\left(31^{\circ} \mathrm{C}\right.$ de temperatura ambiental y $78 \%$ de humedad relativa). El horario establecido fue definido para demostrar la variación de las funciones termorreguladoras en horas frescas y en horas de altas temperaturas, que estarían causando estrés calórico en los animales.

Las mediciones se realizaron en forma semanal en el mes de abril. La temperatura ambiental y la humedad relativa fueron registradas con un termohigrómetro digital LED - HE2 $18 \mathrm{~A} \pm 0.125^{\circ} \mathrm{C}$ y la humedad $\mathrm{al} \pm 1.5 \%$. El registro se hizo cada 5 minutos durante el tiempo que demandó cada muestreo y se obtuvo el promedio. La temperatura rectal se determinó con un termómetro digital, la frecuencia cardiaca (latidos/min) de determinó con un estetoscopio y la frecuencia respiratoria mediante el número de movimientos del flanco por minuto (inspiración y espiración por minuto) por observación directa del animal.

Para la determinación de hematología básica se consideró la hemoglobina $(\mathrm{g} / \mathrm{dl}) \mathrm{y}$ el hematocrito (\%). Además se determinaron las siguientes constantes fisiológicas:

- Volumen corpuscular medio (VCM, fl): [hematocrito (\%) / recuento total de glóbulos rojos (millones $/ \mu \mathrm{l})] * 10$

- Hemoglobina corpuscular media (HCM, pg): [hemoglobina $(\mathrm{g} / \mathrm{dl}) /$ recuento de glóbulos rojos (millones/ $\mu 1)] * 10$

- Concentración de hemoglobina corpuscular media (CHCM, g/dl): [hemoglobina $(\mathrm{g} / \mathrm{dl}) /$ hematocrito $(\%)] * 100$ 
Cuadro 1. Efecto del horario (mañana/tarde) sobre las funciones termorreguladoras de vacas Nellore y cruces con Brown Swiss en condiciones de trópico (Satipo, Perú)

\begin{tabular}{lcccc}
\hline & \multicolumn{2}{c}{ Sin estrés calórico } & \multicolumn{2}{c}{ Con estrés calórico $^{1}$} \\
\cline { 2 - 5 } & Cruzadas & Nellore & Cruzadas & Nellore \\
\hline $\begin{array}{l}\text { Temperatura rectal }\left({ }^{\circ} \mathrm{C}\right) \\
\text { Frecuencia cardiaca } \\
\text { (latidos / minuto) }\end{array}$ & $37.18 \pm 0.72^{\mathrm{a}}$ & $37.61 \pm 0.25^{\mathrm{a}}$ & $39.1 \pm 0.22^{\mathrm{b}}$ & $38.83 \pm 0.46^{\mathrm{b}}$ \\
$\begin{array}{l}\text { Frecuencia respiratoria } \\
\text { (ciclos / minuto) }\end{array}$ & $21.5 \pm 1.22^{\mathrm{a}}$ & $21.67 \pm 1.2^{\mathrm{a}}$ & $42.83 \pm 1.72^{\mathrm{b}}$ & $39.67 \pm 3.7^{\mathrm{b}}$ \\
\hline
\end{tabular}

${ }^{1}$ Mañanas: 06:00-07:00 (18 ㅇ, 80\% Hㅇ); Tardes: 14:00-15:00 (31 ㄷ, 78\% Hㅇ)

$a, b$ Letras diferentes dentro de filas indican diferencias entre grupos $(p<0.05)$

Las muestras fueron colectadas después de la medición de las constantes fisiológicas y por una sola vez en el mes de abril a las 14:00 horas (hora de estrés calórico), mediante punción de la vena yugular y colectadas en tubos Vacutainer con anticoagulante $(5 \mathrm{ml})$. Se evitó estresar a los animales durante la toma de las muestras de sangre. Estas fueron refrigeradas y transportadas al laboratorio de la Facultad de Ciencias Agrarias Sede - Satipo para su análisis utilizando el analizador hematológico Rayto - 7300 (Taiwán).

Las variables de las funciones termorreguladoras fueron analizadas mediante un diseño completamente al azar con arreglo factorial de 2 × 2 (dos horarios y dos grupos raciales). Adicionalmente, se realizó una prueba de comparación de medias. El paquete estadístico utilizado fue SAS v. 9.0. Las variables hematológicas fueron analizadas mediante una prueba de «t $»$ a un nivel de significación de 0.05 , usando el programa SPSS.

\section{Resultados}

La temperatura corporal, la frecuencia cardiaca y la frecuencia respiratoria fueron significativamente diferentes entre el horario de la mañana con el horario de la tarde, tanto en las vacas Nellore como en las cruzadas
( $\mathrm{p}<0.05$; Cuadro 1). Por otro lado, los valores de las funciones termorreguladoras en el horario de escaso o nulo estrés calórico fueron estadísticamente similares entre las vacas cruzadas y las vacas Nellore. En forma similar, tampoco hubo diferencia entre grupos raciales en las horas de estrés calórico; sin embargo, hubo un efecto de la interacción entre la presencia de estrés calórico y el tipo $\operatorname{racial}(\mathrm{p}<0.05$; Cuadro 1$)$.

Los valores hematológicos fueron similares entre las vacas Nellore y sus cruces, excepto para el VCM (Cuadro 2).

\section{Discusión}

Las diferencias encontradas entre las constantes fisiológicas en los horarios de la mañana y la tarde demuestran el efecto de la temperatura ambiental sobre las vacas del estudio. Estas variaciones entre los dos horarios confirman lo manifestado por Alzina et al. (2001), quienes mencionan que el animal pone en marcha el proceso de disipación térmica evaporativa como compensación a la reducida eficiencia de pérdida de calor sensible, lo que le permite mantener la temperatura corporal dentro de los límites fisiológicos. Según CAMS (2014) la estación meteorológica de la Facultad de Ciencias Agrarias 
Cuadro 2. Hematología básica en vacas Nellore y sus cruces con Brown Swiss, criadas en condiciones de trópico (Satipo, Perú)

\begin{tabular}{lccc}
\hline & Cruzadas & Nellore & $\begin{array}{c}\text { Valores } \\
\text { referenciales }^{1}\end{array}$ \\
\hline $\begin{array}{lcc}\text { Hematocrito (\%) } \\
\text { Hemoglobina (g/dl) }\end{array}$ & $35.0 \pm 4.2^{\mathrm{a}}$ & $34.0 \pm 1.4^{\mathrm{a}}$ & $35.1 \pm 7.2$ \\
$\begin{array}{l}\text { Volumen corpuscular medio } \\
\begin{array}{l}\text { (VCM, fl) } \\
\text { Hemoglobina corpuscular media }\end{array}\end{array}$ & $55.48 \pm 3.83^{\mathrm{b}}$ & $60.12 \pm 6.47^{\mathrm{a}}$ & $37.24 \pm 8,40$ \\
$\begin{array}{l}\text { (HCM, pg) } \\
\begin{array}{l}\text { Concentración de hemoglobina } \\
\text { corpuscular media (CHCM, g/dl) }\end{array}\end{array}$ & $34.78 \pm 0.43^{\mathrm{a}}$ & $34.69 \pm 0.21^{\mathrm{a}}$ & $38.97 \pm 3.62$ \\
\hline
\end{tabular}

$a, b$ Letras diferentes dentro de filas indican diferencias entre razas $(p<0.05)$

${ }^{1}$ Rango de referencia para vacas Bos indicus (Moreno et al., 2008)

registró en Rio Negro, Satipo, temperatura ambiental mínima de $17.96 \pm 1.03$, y máxima de $34.11 \pm 0.61$, humedad relativa de $81.22 \%$ y precipitación de $116 \pm 49 \mathrm{~mm}$ para el mes de abril en los años 2010-2014.

Baccari (2001) menciona que el ganado ante situaciones de estrés térmico usa mecanismos fisiológicos alternativos de pérdida de calor, tales como aumentar la frecuencia respiratoria (taquipnea), sudoración. Así mismo, Espinoza et al (2009) mencionan que la respiración es lo primero que reacciona en el bovino como mecanismo adicional de ventilación pulmonar y evaporación para mantener la temperatura corporal. Ante esta situación el ganado incrementa su frecuencia respiratoria, ventilación pulmonar. Por otro lado, se conoce que cuando las temperaturas ambientales alcanzan los $26.7^{\circ} \mathrm{C}$ en el Bos taurus y $35^{\circ} \mathrm{C}$ en el Bos indicus, esos mecanismos son incapaces de disipar todo el exceso de calor (Espinoza et al., 2011). Los datos del presente estudio demuestran que, incluso con el gran aumento en la frecuencia respiratoria, las vacas no lograron evitar la hipertermia.
Las vacas, tanto las Nellore como las cruzadas presentaron valores similares en cuanto a la termorregulación en horas de nulo estrés calórico, así como también en horas de estrés calórico; por tanto, el factor genético, en este caso, no estaría asociado a una mayor resistencia al estrés calórico (Ferreira et al., 2006). Arias et al. (2008) refieren que el estrés calórico afecta seriamente el sistema neural, inmunológico y endocrino, que conlleva a estados prepatológicos donde convergen otros tipos de factores que comprometen la salud del animal. En ecosistemas tropicales el estrés calórico es un factor importante que afecta la rentabilidad del bovino, de ahí la importancia de comprenderlo para plantear estrategias de producción sostenible.

Las vacas presentaron valores de hematocrito y de hemoglobina casi al límite de los valores de referencia, pero dentro de los rangos aceptables, lo que permite suponer que los animales están regulando su estado fisiológico. A pesar de no hallarse diferencias entre los tipos raciales, el VCM y la HCM estuvieron por encima de los rangos referenciales. El primero relacionado al in- 
cremento en el tamaño de los glóbulos rojos y el segundo en la cantidad de hemoglobina contenida en un glóbulo rojo, lo que puede deberse al incremento de la actividad metabólica necesaria para satisfacer las demandas energéticas, bajo condiciones de estrés y como consecuencia de una mayor demanda de oxígeno (Ribeiro et al., 2018).

\section{Literatura Citada}

1. Alzina A, Farfán J, Valencia E, Yokoyama J. 2001. Condición ambiental y su efecto en la temperatura rectal y frecuencia respiratoria en bovinos cruzados (Bos taurus x Bos indicus) del estado de Yucatán, México. Rev Biomed 12: $112-121$.

2. Araúz E, Fuentes A, Mendez N. 2010. Alteración diurna de la carga calórica corporal e interrelación de las temperaturas rectal y láctea en vacas cruzadas (6/8 Bos taurus x 2/8 Bos indicus), Pardo Suizo y Holstein bajo estrés calórico diurno durante la época seca en el clima tropical húmedo. REDVET 11(11). [Internet]. Disponible en: https:// www.redalyc.org/articulo.oa?id$=636169-33002-\& \mathrm{idp}=1 \& \mathrm{cid}=1024518$

3. Arias RA, Mader TL. 2011. Environmental factors affecting daily water intake on cattle finished in feedlots. $\mathrm{J}$ Anim Sci 89: 245-51. doi: 10.2527/ jas.2010-3014.

4. Arias RA, Mader TL, Escobar PC. 2008. Factores climáticos que afectan el desempeño productivo del ganado bovino de carne y leche. Arch Med Vet 40: 7-22. doi: 10.4067/S0301-732X2008000100002

5. Baccari Jr F, Valle R, Baccari F, Menezes WF, Slansky F, Williams FL, Ross FA, et al. 2001. Manejo ambiental da vaca leiteira em climas quentes. Brasil: Londrina. 142 p.
6. [CAMS] Comisión Ambiental Municipal Satipo. 2014. Diagnóstico ambiental local de la provincia de Satipo. Disponible en: http://www.devida.gob.pe/ docu-ments/20182/332409/DAL_Satipo2014/21594873-deb8-4a7d-b444$\overline{5} 811 \mathrm{ef} 4666 \mathrm{e} 6$

7. Díaz E, Narváez-Solarte W, Giraldo J. 2016. Alteraciones hematológicas y zootécnicas del pollo de engorde bajo estrés calórico. Info Tecnol 27: 221-230. doi: 10.4067/S0718-07642016000300021

8. Espinoza J, Ortega R, Palacios A, Guillén A. 2011. Tolerancia al calor y humedad atmosférica de diferentes grupos raciales de ganado bovino. Rev MVZ Córdova 16: 2302-2309. doi: 10.21897/rmvz.288

9. Espinoza JL, Sánchez J, Gracia JA, Sánchez JR, Ortega R, Palacios A. 2009. Thermoregulation differs in Chinampo (Bos taurus) and locally born dairy cattle. Turk J Vet Anim Sci 33: 175180. doi: 10.3906/vet-0704-24

10. Ferreira F, Pires M, Martínez M, Coelho S, Carvalho A, Ferreira P, Facury E, Campos W. 2006. Parâmetros fisiológicos de bovinos cruzados submetidos ao estresse calórico. Arq Bras Med Vet Zoo 58: 732-738. doi: 10.1590/S0102-09352006000500005

11. Hansen PJ. 2004. Physiological and cellular adaptations of zebu cattle to thermal stress. Anim Reprod Sci 82-83: 349-360. doi: 10.1016/j.anireprosci.2004.04.011

12. Klein B. 2014. Cunningham Fisiología Veterinaria. $5^{\circ}$ ed. España: Elsevier. $624 \mathrm{p}$.

13. Moreno F, Builes J, Cadavid J. 2008. Evaluación de 30 parámetros hemáticos en bovinos Bos indicus en los Municipios de San Juan de Urabá y Arboletes del Uraba Antioqueño. Universidad CES. Colombia. [Internet]. Disponible en: http:/ /bdigital.ces.edu.co:8080/jspui/bitstream/ 10946/1000/1/Evalua-cion_parametros_hemaliticos.pdf 
14. Olkowski AA, Duke T, Wojnarowicz C. 2005. The aetiology of hypoxaemia in chickens selected for rapid growth. Comp Biochem Physiol A 141: 122-131. doi: 10.1016/j.cbpb.2005.04.011

15. Ribeiro NM, Ribeiro LN, Bozzi R, Costa GR. 2018. Physiological and biochemical blood variables of goats subjected to heat stress - a review. J Appl Anim Res 46: 1036-1041. doi: 10.1080/09712119.2018.1456439

16. Roca A. 2011. Efecto del estrés calórico en el bienestar animal, una revisión en tiempo de cambio climático. Espamciencia 2: 15-25. 